\title{
Serotype IV Sequence Type 468 Group B Streptococcus Neonatal Invasive Disease, Minnesota, USA
}

\section{Sarah Teatero, Patricia Ferrieri, Nahuel Fittipaldi}

To further understand the emergence of serotype IV group B Streptococcus (GBS) invasive disease, we used wholegenome sequencing to characterize 3 sequence type 468 strains isolated from neonates in Minnesota, USA. We found that strains of tetracycline-resistant sequence type 468 GBS have acquired virulence genes from a putative clonal complex 17 GBS donor by recombination.

$\mathrm{G}$ roup B Streptococcus (GBS) is an opportunistic pathogen responsible for infections in neonates and adults; infection results in substantial morbidity and mortality worldwide (1). Ten serotypes (Ia, Ib, II-IX) have been described on the basis of their capsular polysaccharide antigen (2). A widely used multilocus sequence typing (MLST) scheme enables discrimination of GBS strains in $>700$ sequence types (STs), which are grouped in a few clonal complexes (CCs) (3).

We recently reported increased frequency of isolation of both carriage and invasive serotype IV GBS in Minnesota, USA, and increased frequency of isolation of serotype IV strains causing invasive disease in 3 Canadian provinces (4-7). We also showed that emerging serotype IV GBS organisms are genetically heterogeneous. Although most serotype IV strains belonged to ST459 (CC1) or ST452 (CC23), the population also comprised ST3, ST196, ST710, ST711, ST291, ST682, and ST468 isolates, the latter a single-locus variant of ST452 (4-7). Little is known about several of these less common serotype IV STs causing human disease, including their genomic makeup and antimicrobial drug resistance profiles. Also unknown are the molecular mechanisms underlying their emergence.

Homologous recombination occurs frequently in GBS and can involve vast areas of the genome in some lineages $(8,9)$. Although MLST does not permit detection of recombination events with accuracy, the use of now relatively inexpensive next-generation wholegenome sequencing (WGS) has enabled the study of

Author affiliations: Public Health Ontario, Toronto, Ontario,

Canada (S. Teatero, N. Fittipaldi); University of Minnesota Medical School, Minneapolis, Minnesota, USA (P. Ferrieri); University of Toronto, Toronto (N. Fittipaldi)

DOI: http://dx.doi.org/10.3201/eid2211.152031 recombination in GBS at the population level $(4,9,10)$. We used WGS analysis of 3 ST468 isolates recovered from neonates in Minnesota to test the hypothesis that recombination is a main driver of genetic diversity among serotype IV GBS.

\section{The Study}

We studied 3 ST468 strains, 2 isolated in 2007 and 1 in 2010 from neonates with early-onset or late-onset GBS disease (Table). We prepared genomic DNA by using a QIAGEN DNA MiniKit (QIAGEN, Toronto, ON, Canada) and genomic libraries by using Nextera XT kits (Illumina, San Diego, CA, USA). We sequenced the libraries as pairedend reads (150 bp $+150 \mathrm{bp})$ by using a MiSeq instrument (Illumina). We determined pili content in silico by aligning short-read WGS data with the sequences of pilus island (PI) 1, PI-2a, and PI-2b and using MOSAIK (5). The 3 ST468 strains possessed PI-2b only.

We next aligned the short reads to the genome of serotype IV ST452 strain NGBS572 (GenBank accession no. CP007632.1) and identified polymorphisms by using a variant ascertainment algorithm as previously described (4). The 3 ST468 strains differed from the ST452 reference strain by an average of 725 single-nucleotide polymorphisms (SNPs). When we plotted genome-wide SNPs of the ST468 strains against the genome of the reference strain (using BRIG [11]), we found a conspicuous area with an overabundance of SNPs (positions 210-320 kbp of the reference genome [Figure 1]). Nonrandom polymorphism distribution suggests recombination. To confirm this hypothesis, we analyzed the polymorphism data with BRAT NextGen (12), run with 20 iterations, 100 replicates, and a significance cutoff of 0.05 . The analysis defined a region of recombination in strain PF-10 corresponding to positions 211,553-331,548 bp of the reference genome (Figure 1). A slightly narrower area of recombination was defined in strains PF-17 and PF-18 (211,553-323,601 and 249,415323,601 , respectively) (Figure 1).

In all 3 strains, the gene $t k t$, used in the MLST scheme, was found within the region of recombination, which explained the MLST result that ST468 is a single-locus variant of ST452. The common region of recombination also contained genes involved in metabolic pathways, such as nagA, encoding an $\mathrm{N}$-acetylglucosamine-6-phosphate deacetylase involved in sialic acid metabolism. The ability of certain 
Table. Strains of neonatal invasive group B Streptococcus disease used in genetic diversity study, Minnesota, USA*

\begin{tabular}{lccccc}
\hline Isolate no. & Year cultured & Culture source & Patient age, & SNPs to NGBS572 & SRA accession no. \\
\hline PF-10 & 2010 & Blood & 0 & 766 & SRR4101407 \\
PF-17 & 2007 & Blood & 2 & 706 & SRR4101408 \\
PF-18 & 2007 & Blood & 9 & 704 & SRR4101409 \\
\hline${ }^{*}$ All isolates were serotype IV (6). SNP, single-nucleotide polymorphism; SRA, Sequence Read Archive. & \\
\hline
\end{tabular}

streptococcal species to use sialic acid might play a role in the persistence and survival of these infecting organisms in vivo (13). Additionally, the region contained genes encoding the glycerol kinase GlpK, the aminopeptidase PepC, and the NAD synthetase nicotinamide adenine dinucleotide. This region also contains a bacteriocin (enterocin A) and penicillin-binding proteins PBP2X and PBP1A.

To identify the potential recombination donor, we first built a pseudoreference genome of strain PF-10. In brief, we performed de novo assembly of short reads generated for this strain as previously described (5), ordered obtained contigs against the reference genome of the ST452 strain NGBS572 with progressiveMauve (14), and concatenated the contigs with a separator that introduced a stop codon in every reading frame. Next, we aligned whole-genome sequence short reads of strains belonging to 12 different STs against the PF-10 pseudogenome and identified polymorphisms as described here. These $12 \mathrm{STs}$ represent the
6 major CCs most frequently associated with GBS disease in humans (9). The pattern of polymorphisms strongly suggested that the donor of the recombined fragment was a CC17 strain (online Technical Appendix Figure, http:// wwwnc.cdc.gov/EID/article/22/11/15-2031-Techapp1. pdf). Strains of $\mathrm{CC} 17$ are associated with neonatal infections, especially late-onset disease (15).

Lateral exchange of antimicrobial drug resistance mediated by mobile genetic elements occurs frequently in GBS. The implications of these events (beyond the obvious gain of resistance to a particular antimicrobial agent) are not yet fully understood. For example, Da Cunha et al. recently presented convincing evidence that tetracycline resistance has contributed to the worldwide expansion of GBS clones causing disease in humans (9). Strains of one of the major STs associated with serotype IV GBS invasive disease, ST452, are sensitive to tetracycline (5). Our unpublished observations suggest that the genome of

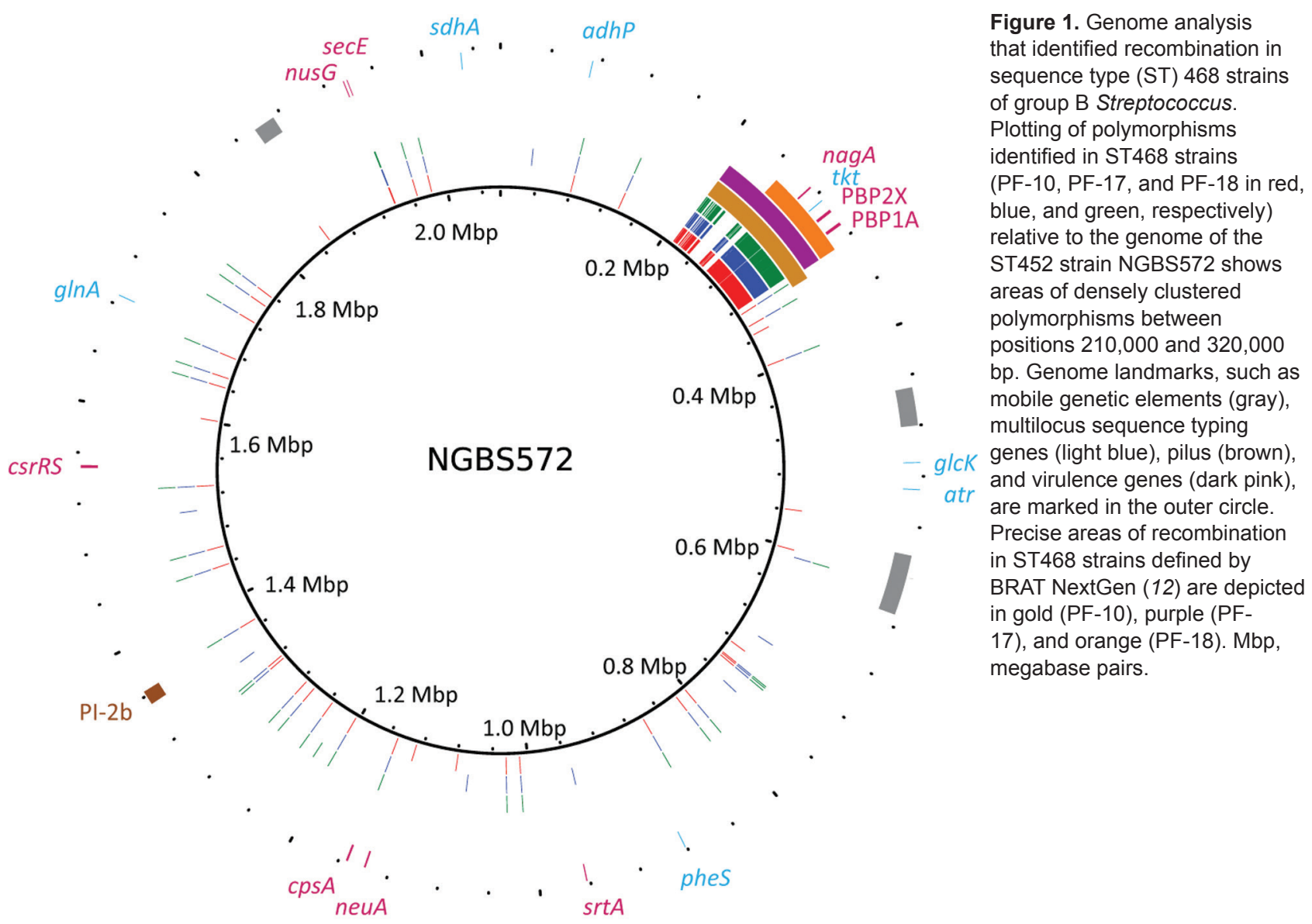




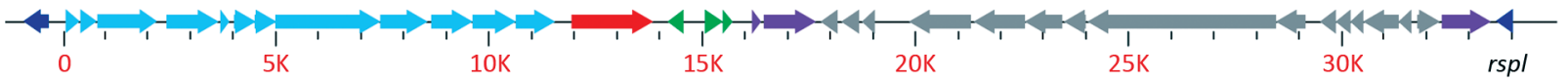

Hypothetical protein

Figure 2. Representative diagram of a tetM-containing mobile genetic element (MGE) in group B Streptococcus sequence type (ST) 468 strains. Genome analysis discovered that the MGE is integrated at the 3' end of gene rspl. Genes common between ST468 strains and the ST452 strain NGBS572 are shown in dark blue. Genes involved in conjugation are shown in light blue, regulatory genes in green, recombination genes in purple, and the gene encoding tetracycline resistance (tetM) in red. Cis integrative and mobilizable element genes (CIME) are shown in gray. Mbp, megabase pairs

ST452 strains could be considered a hybrid genome combining sequences of ST23 and ST17. ST468 strains analyzed here have additional ST17 content and were resistant to tetracycline. We determined that the genomes of all 3 ST468 strains contained gene tet $M$, which was carried on a mobile genetic element (MGE) (Figure 2). Areas of this MGE have $99 \%$ identity (blastn, www.ncbi.nlm.nih. gov/blast/Blast.cgi) to transposon Tn916. The Tn916-like element lies upstream of cis-mobilizable genetic content. Further inspection revealed that the MGE is inserted in the $3^{\prime}$ end of gene rspI (positions 232,722-250,539 bp of the ST452 genome), which is a known hot spot for integrative conjugative element integration in GBS (8).

\section{Conclusions}

WGS-based studies are expanding our understanding of genome reshaping in GBS and beginning to shed light on the population dynamics of this species. In particular, the use of WGS has shown that strains with multiple genome backgrounds are involved in the emergence of serotype IV GBS disease in North America $(5,6)$. Here, we show that strains of one of these diverse backgrounds, ST468, have acquired additional genetic material from a neonatal-associated lineage by means of recombination. We also show that these isolates have acquired tetracycline resistance by lateral transfer. The changing epidemiology of GBS disease, with the involvement of a diverse array of strain genotypes, warrants continuing monitoring of colonization and invasive serotype IV GBS infections. Our data also support including this serotype in GBS vaccine formulations currently under development.

\section{Acknowledgments}

We thank staff at Public Health Ontario Genome Core for Illumina sequencing of our strains and Aurea E. Flores for serotyping these strains.

This work was supported by Public Health Ontario.
Ms. Teatero is a research associate at Public Health Ontario. Her primary research interest is the molecular epidemiology of group B Streptococcus.

\section{References}

1. Le Doare K, Heath PT. An overview of global GBS epidemiology. Vaccine. 2013;31(Suppl 4):D7-12. http://dx.doi.org/10.1016/j. vaccine.2013.01.009

2. Cieslewicz MJ, Chaffin D, Glusman G, Kasper D, Madan A, Rodrigues S, et al. Structural and genetic diversity of group B Streptococcus capsular polysaccharides. Infect Immun. 2005;73: 3096-103. http://dx.doi.org/10.1128/IAI.73.5.3096-3103.2005

3. Jones N, Bohnsack JF, Takahashi S, Oliver KA, Chan MS, Kunst F, et al. Multilocus sequence typing system for group B Streptococcus. J Clin Microbiol. 2003;41:2530-6. http://dx.doi.org/10.1128/JCM.41.6.2530-2536.2003

4. Teatero S, Athey TB, Van Caeseele P, Horsman G, Alexander DC, Melano RG, et al. Emergence of serotype IV group B Streptococcus adult invasive disease in Manitoba and Saskatchewan, Canada, is driven by clonal sequence type 459 strains. J Clin Microbiol. 2015;53:2919-26. http://dx.doi.org/10.1128/JCM.01128-15

5. Teatero S, McGeer A, Li A, Gomes J, Seah C, Demczuk W, et al. Population structure and antimicrobial resistance of invasive serotype IV group B Streptococcus, Toronto, Ontario, Canada. Emerg Infect Dis. 2015;21:585-91. http://dx.doi.org/10.3201/ eid2014.140759

6. Ferrieri P, Lynfield R, Creti R, Flores AE. Serotype IV and invasive group B Streptococcus disease in neonates, Minnesota, USA, 20002010. Emerg Infect Dis. 2013;19:551-8. http://dx.doi.org/10.3201/ eid1904.121572

7. Diedrick MJ, Flores AE, Hillier SL, Creti R, Ferrieri P. Clonal analysis of colonizing group B Streptococcus, serotype IV, an emerging pathogen in the United States. J Clin Microbiol. 2010;48:3100-4. http://dx.doi.org/10.1128/JCM.00277-10

8. Brochet M, Couvé E, Glaser P, Guedon G, Payot S. Integrative conjugative elements and related elements are major contributors to the genome diversity of Streptococcus agalactiae. J Bacteriol. 2008;190:6913-7

9. Da Cunha V, Davies MR, Douarre PE, Rosinski-Chupin I, Margarit I, Spinali S, et al.; DEVANI Consortium. Streptococcus agalactiae clones infecting humans were selected and fixed through the extensive use of tetracycline. Nat Commun. 2014;5:4544. http://dx.doi.org/10.1038/ncomms5544

10. Flores AR, Galloway-Peña J, Sahasrabhojane P, Saldaña M, Yao H, Su X, et al. Sequence type 1 group B Streptococcus, an 
emerging cause of invasive disease in adults, evolves by small genetic changes. Proc Natl Acad Sci U S A. 2015;112:6431-6. http://dx.doi.org/10.1073/pnas.1504725112

11. Alikhan NF, Petty NK, Ben Zakour NL, Beatson SA. BLAST Ring Image Generator (BRIG): simple prokaryote genome comparisons. BMC Genomics. 2011;12:402. http://dx.doi.org/10.1186/14712164-12-402

12. Marttinen P, Hanage WP, Croucher NJ, Connor TR, Harris SR, Bentley SD, et al. Detection of recombination events in bacterial genomes from large population samples. Nucleic Acids Res. 2012;40:e6. http://dx.doi.org/10.1093/nar/gkr928

13. Choi CW, An HY, Lee YJ, Lee YG, Yun SH, Park EC, et al. Characterization of Streptococcus pneumoniae $\mathrm{N}$-acetylglucosamine-6-phosphate deacetylase as a novel diagnostic marker. J Microbiol. 2013;51:659-64. http://dx.doi.org/10.1007/s12275-013-3451-8

14. Darling AE, Mau B, Perna NT. progressiveMauve: multiple genome alignment with gene gain, loss and rearrangement. PLoS One. 2010;5:e11147. http://dx.doi.org/10.1371/journal.pone.0011147

15. Manning SD, Springman AC, Lehotzky E, Lewis MA, Whittam TS, Davies HD. Multilocus sequence types associated with neonatal group B streptococcal sepsis and meningitis in Canada. J Clin Microbiol. 2009;47:1143-8. http://dx.doi.org/10.1128/JCM.01424-08

Address for correspondence: Nahuel Fittipaldi, Public Health Ontario, Toronto Laboratories, 661 University Ave, Ste 17-100, Toronto, ON M5G 1M1, Canada; email: nahuel.fittipaldi@oahpp.ca

\section{June 2015: Bacterial/Fungal Infections}

- Sequence Type 4821 Clonal Complex Serogroup B Neisseria meningitidis in China, 1978-2013

- Estimated Deaths and Illnesses Averted

During Fungal Meningitis Outbreak Associated with Contaminated Steroid Injections, United States, 2012-2013

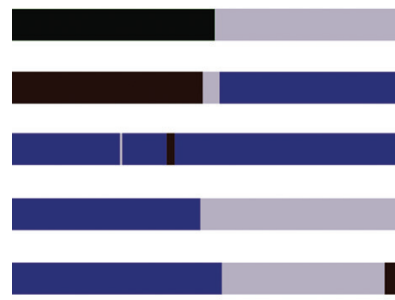

- Global Burden of Invasive Nontyphoidal Salmonella Disease, 2010

- Dose-Response Relationship between Antimicrobial Drugs and Livestock-associated MRSA in Pig Farming

- Cost-effectiveness of Chlamydia Vaccination Programs for Young Women

- Mycobacterium bovis in Panama, 2013

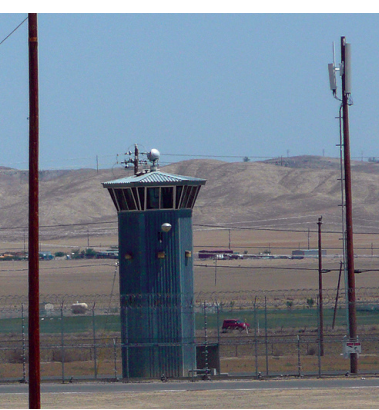

- Hospitalization Frequency and Charges for Neurocysticercosis, United States, 2003-2012

- Additional Drug Resistance of MultidrugResistant Tuberculosis in Patients in 9 Countries

- Oral Cholera Vaccination Coverage, Barriers to Vaccination, and Adverse Events following Vaccination, Haiti, 2013

- Endemic Melioidosis in Residents of Desert Region after Atypically Intense Rainfall in Central Australia, 2011

- Invasion Dynamics of White-Nose Syndrome Fungus, Midwestern United States, 2012-2014

- Coccidioides Exposure and Coccidioidomycosis among Prison Employees, California, United States
- Prospective Multicenter International Surveillance of Azole Resistance in Aspergillus fumigatus

- Oligoarthritis Caused by Borrelia bavariensis, Austria, 2014

- European Rabbits as Reservoir for Coxiella burnetii

- Drug ResistanceAssociated Mutations in Mycoplasma genitalium in Female Sex Workers, Japan

- Lack of Protection Against Ebola Virus from Chloroquine in Mice and Hamsters

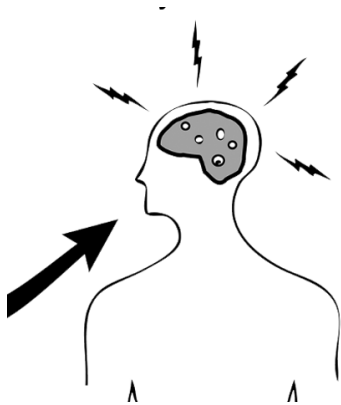

- Wohlfahrtiimonas chitiniclastica Bacteremia Associated with Myiasis, United Kingdom

- Response to Dectection of New Delhi Metallo$\beta$-Lactamase-Producing Bacteria, Brazil
- Histoplasmosis in Idaho and Montana, USA, 2012-2013

- Seroconversions to Rickettsiae in US Military Personnel in South Korea

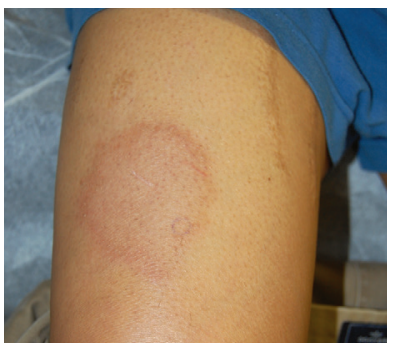

- MRSA spa †1081, a Highly Transmissible Strain Endemic to Hong Kong, China, in the Netherlands

- Multibacillary Leprosy in an Active Duty Military Member

- Tickborne Relapsing Fever in Southern Iran, 2011-2013

- Reducing the Risk for Waterborne Nosocomial Neonatal Legionellosis

- Carnobacterium divergens Bacteremia in Woman

- Fatal Nosocomial MDR TB Identified through Routine Genetic Analysis and Whole-Genome Sequencing
EMERGING INFECTIOUS DISEASES http://wwwnc.cdc.gov/eid/articles/ issue/2 1/06/table-of-contents 